\title{
Controle de um conversor formador de rede trifásico funcionando em modo isolado
}

\author{
Renata Cristina da Silva* Seleme I. Seleme Jr** \\ * Programa de Pós-Graduação em Engenharia Elétrica - Universidade \\ Federal de Minas Gerais - Av. Antônio Carlos 6627, 31270-901, Belo \\ Horizonte, $M G$, Brasil (e-mail: renatacristina@ymail.com) \\ ** Departamento de Engenharia Eletrônica, Universidade Federal de \\ Minas Gerais - Av. Antônio Carlos 6627, 31270-901, Belo Horizonte, \\ MG,Brasil(e-mail: seleme@cpdee.ufmg.br)
}

\begin{abstract}
This article presents the control of a three-phase converter as a voltage source to be applied in microgrids in island mode. The converter works as a grid-forming, ie it imposes frequency and voltage at the common coupling point (PCC) of the microgrid. The converter consists of a LCL filter, three-phase DC/AC converter and an bidirectional interleaved DC/DC converter and battery power storage. The control is implemented in synchronous reference frame and used to control the current and voltage at the PCC . The DC link voltage is controlled by the bidirectional DC/DC converter with voltage and current loop. The system is simulated in MATLAB / Simulink, by which the operation of the circuit is proven.

Resumo: Este artigo apresenta o controle de um conversor trifásico controlado como fonte de tensão para ser aplicado em microrredes no modo de funcionamento isolado da rede. $\mathrm{O}$ conversor trabalha como formador de rede, ou seja, impõe a frequência e a tensão no ponto de acoplamento comum (PAC) da microrrede. O conversor é composto por filtro LCL, conversor $\mathrm{CC} / \mathrm{CA}$ de trifásico, conversor $\mathrm{CC} / \mathrm{CC}$ bidirecional entrelaçado e armazenamento de energia em baterias. É implementado um controle em referencial síncrono da corrente e da tensão no PAC. A tensão no link CC é controlada pelo conversor CC/CC bidirecional, com malha de tensão e corrente. O sistema é simulado no MATLAB/Simulink, pelo qual é comprovado o funcionamento do circuito.
\end{abstract}

Keywords: three-phase converter; grid-forming; PI controller; synchronous reference frame; island mode; bidirecional CC/CC converter

Palavras-chaves: inversor trifásico; formador de rede; controle PI; referencial síncrono; microrrede isolada; conversor $\mathrm{CC} / \mathrm{CC}$ bidirecional

\section{INTRODUÇÃO}

A Agência Nacional de Energia Elétrica ANEEL define a microgeração distribuída como uma fonte de energia de origem renovável com potência de até $75 \mathrm{~kW}$ conectadas na rede de distribuição por meio de instalações consumidoras (ANEEL (2012)). Estas fontes de energia além de atender as necessidades do consumidor, por estarem conectadas ao sistema interligado podem injetar a energia não usada na rede. Com a geração distribuída, surgiu as microrredes de energia. De acordo com o padrão IEEE 1547.4(2001, p. 3, apud Antunes et al. (2016)), define-se microrredes como sendo um conjunto de cargas elétricas e microgeração de energia elétrica operando como um único sistema de energia.

Em uma microrrede, os inversores podem ser classificados de acordo com a sua função em formadores de rede, supridor de rede e de suporte a rede (Rocabert et al. (2012)). Em relação ao modo de funcionamento, esta pode funci-

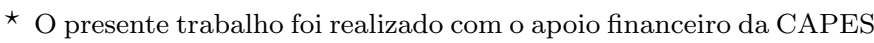
- Brasil
}

onar conectada a rede principal ou em modo isolado. No modo de operação conectado ao sistema principal, ou rede, esta impõe o nível de tensão e frequência na microrrede. No modo isolado, ou seja, não conectado a rede, a microrrede fica sem a referência de tensão e frequência para as suas unidades geradoras. Assim é necessário implementar, nas microrredes que operam de forma isolada, o conversor formador de rede. Este conversor funcionando como fonte de tensão ideal, será responsável por estabelecer a tensão e frequência da microrrede no caso de ausência da rede principal (de Matos et al. (2014)). O conversor de supridor de rede é o que faz a interface entre a fonte de energia renovável e a microrrede. Como seu nome sugere, a sua função é suprir a rede com a potência vinda da sua fonte de energia. O conversor de suporte a rede é projetado para funções que tem como objetivo melhorar a qualidade da energia da microrrede. Estes serviços podem incluir o controle de reativos, regulação de tensão e frequência (Volt/Var, Volt/Watt) (Araújo et al. (2017)). Este trabalho irá focar no projeto e modelagem de um conversor funcionando como fonte de tensão com armazenamento de energia por baterias para atuar como formador de rede. $\mathrm{O}$ 


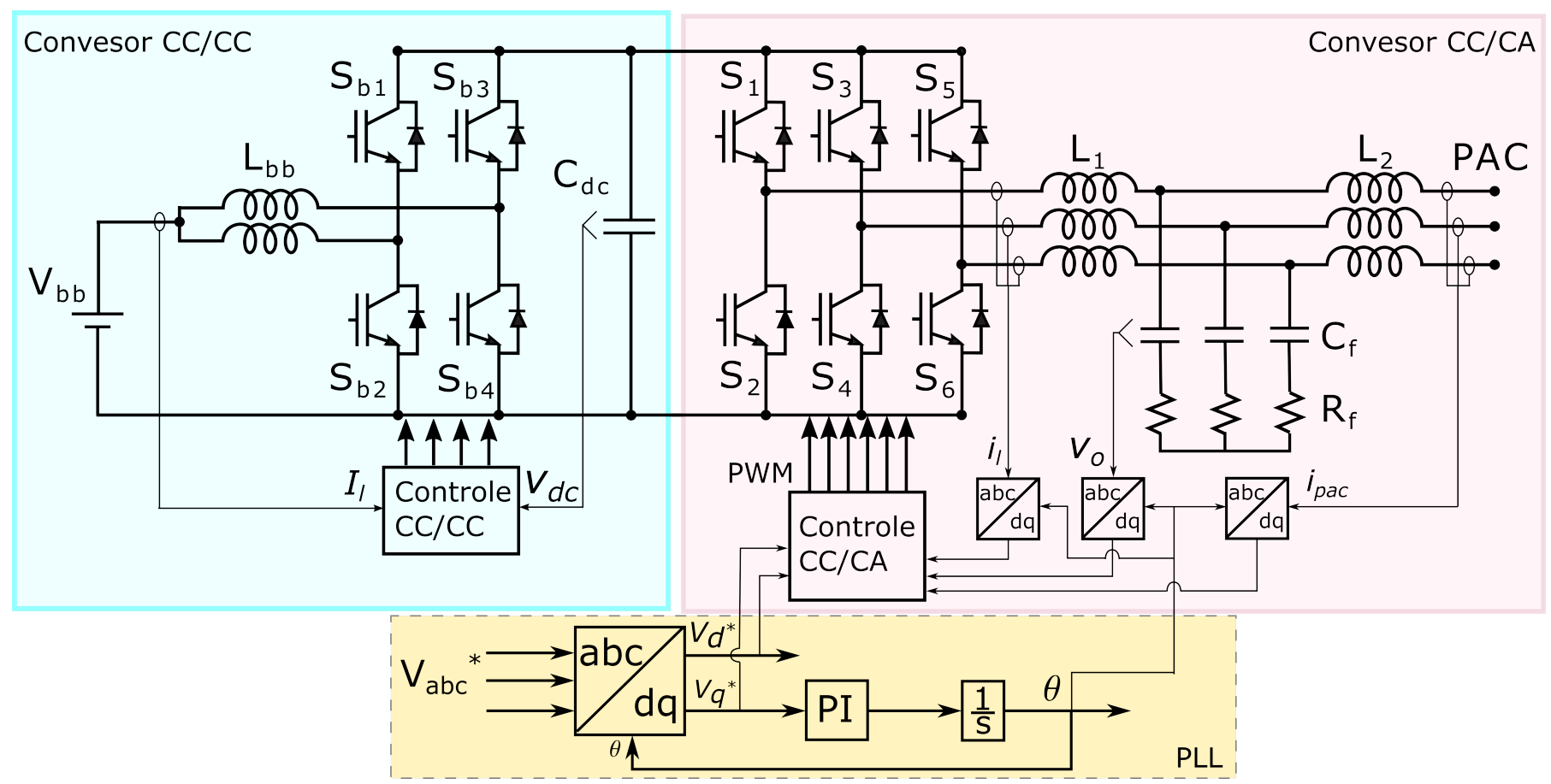

Figura 1. Sistema implementado

conversor fonte de tensão (VSC - voltage source converter) é composto por um conversor do $\mathrm{CC} / \mathrm{CA}$, um conversor $\mathrm{CC} / \mathrm{CC}$ bidirecional e entrelaçado para a carga e descarga do banco de baterias e manutenção da tensão no barramento CC. Os dois sistemas serão modelados separadamente. O conversor do lado da rede é um inversor $\mathrm{CC} / \mathrm{CA}$ trifásico e é responsável por controlar a transferência de energia ativa e reativa para a rede. $\mathrm{O}$ conversor formador de rede é baseado em controle em referencial síncrono com duas malhas de controle, uma de corrente e outra de tensão (Antunes et al. (2016), Rocabert et al. (2012), Bian et al. (2017)). Para gerar a referência de entrada, utilizou-se um referência trifásica que foi transformado para referencial síncrono, gerando a referência de tensão do ponto de acoplamento comum (PAC) no eixo d e q. A malha externa de controle regula a tensão no PAC e a malha interna regula a corrente fornecida pelo conversor. Normalmente, para conversores operando conectado a rede, a tensão do barramento CC é controlada pelo conversor (Teodorescu et al. (2011)), mas para operação isolada a tensão em cima do capacitor do barramento é controlada pelo elemento que armazena energia (Singh et al. (2018)). Assim, para o conversor $\mathrm{CC} / \mathrm{CC}$ foi implementado um controle em cascata. A malha externa de tensão regula a tensão no barramento CC, e uma malha interna regula a corrente nos indutores, que é a responsável pela transferência de potência da fonte CC para o barramento (Serban and Ion (2017)).

\section{SISTEMA PROPOSTO}

Propõe-se para o sistema do formador de rede, a configuração da figura 1 , com o conversor $\mathrm{CC} / \mathrm{CA}$, o conversor bidirecional $\mathrm{CC} / \mathrm{CC}$, bateria e filtro LCL para conexão com a rede. Os parâmetros do sistema são apresentados na tabela 1 .
Tabela 1. Parâmetros do sistema

\begin{tabular}{c|c} 
Parâmetros & Valor \\
\hline Potência Nominal & $10 \mathrm{kVA}$ \\
Tensão Nominal CA & $127 \mathrm{~V}$ \\
Frequência fundamental f & $60 \mathrm{~Hz}$ \\
Tensão DC & $450 \mathrm{~V}$ \\
Capacitância DC & $3060 \mu \mathrm{F}$ \\
Indutância do filtro $L_{1}$ e $L_{2}$ & $2 \mathrm{mH}$ \\
Resistência do indutor $R_{1}$ e $R_{2}$ & $0,015 \Omega$ \\
Capacitância do filtro $C_{f}$ & $20 \mu \mathrm{F}$ \\
Resistência de amortecimento $R_{f}$ & $1,8 \Omega$ \\
Indutância do conversor bidirecional $L_{b b}$ & $3,7 \mathrm{mH}$ \\
Resistência do indutor $r_{b b}$ & $71 \mathrm{~m} \Omega$ \\
Frequência de chaveamento & $3420 \mathrm{~Hz}$ \\
Tensão do arranjo de baterias & $144 \mathrm{~V}$ \\
\hline
\end{tabular}

\subsection{Filtro $L C L$}

O filtro implementado faz a conexão entre o conversor e a rede. A tensão de saída do conversor possui harmônicos na frequência de chaveamento, o que resulta em harmônicos na corrente e tensão da rede. O filtro serve para eliminar os harmônicos do decorrentes do chaveamento.

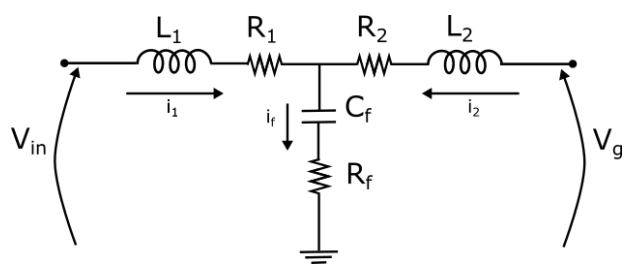

Figura 2. Filtro LCL

O filtro LCL foi escolhido porque ele tem boa atenuação de ripple para baixos valores de indutância. Ele possui como característica atenuação de $60 \mathrm{db} /$ década para frequências acima da frequência de ressonância. Isto possibilita usar baixas frequências de chaveamento juntamente com um 
filtro com frequência de ressonância distante o suficiente da frequência da rede (Kahlane et al. (2014)).

A frequência de ressonância para o filtro pode ser calculada pela equação (1):

$$
f_{\text {res }}=\frac{1}{2 \pi} \sqrt{\frac{L_{1}+L_{2}}{L_{1} L_{2} C_{f}}}
$$

A função de transferência entre a corrente da rede $\left(I_{g}\right)$ e a tensão no conversor (V) do filtro LCL, é descrita pela equação (2):

$$
\frac{I_{g}(s)}{V(s)}=\frac{R_{f} \cdot C_{f} \cdot s+1}{C_{f} \cdot L_{2} \cdot L_{1} \cdot s^{3}+a_{1} s^{2}+a_{2} s+a_{3}}
$$

sendo

$a_{1}=C_{f} L_{1} R_{2}+C_{f} L_{2} R_{1}+C_{f} L_{1} R_{f}+C_{f} L_{2} R_{f}$ $a_{2}=L_{1}+L_{2}+C_{f} R_{1} R_{2}+C_{F} R_{1} R_{F}+C_{f} R_{2} R_{f}$ $a_{3}=R_{1}+R_{2}$

A frequência de ressonância calculada para o filtro deste sistema é de $1125,4 H z$. Sendo a frequência de chaveamento de $3,42 k H Z$, a frequência de ressonância do filtro é suficiente para atenuar os harmônicos proveniente do chaveamento. Como mostra o diagrama de bode do filtro LCL em figura 3, a atenuação depois da frequência de ressonância é suficiente para eliminar as componentes harmônicas da frequência de chaveamento e ainda é consideravelmente mais alta que a frequência da banda de passagem dos controladores, que são projetados para uma década abaixo da frequência de chaveamento.

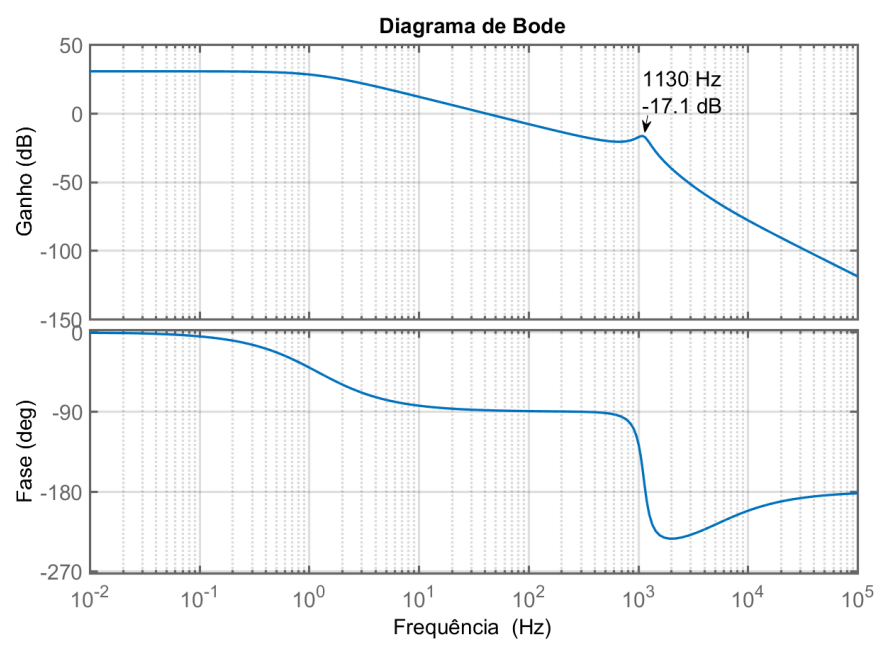

Figura 3. Diagrama de bode do filtro LCL

\subsection{PLL - Phased locked loop}

A PLL (do inglês phased locked loop) são circuitos que geram uma frequência em fase com o sinal de entrada. Geralmente, usados para sincronizar dois circuitos diferentes, por exemplo, o conversor e a rede. Para a geração da referência de frequência foi implementada a SRFPLL (synchronous reference frame PLL) (Teodorescu et al. (2011)). Na SRF-PLL, a tensão trifásica é transformada para o referencial síncrono dq usando a transformada de Park. O ângulo de fase $\theta$ é controlado por uma malha de controle que regula a componente q da tensão em zero. O ângulo de fase gerado é então usado nas transformações de referencial, garantindo que a tensão trifásica gerada tenha a mesma frequência da rede. Como o inversor, trabalha isolado da rede, a referência de entrada da PLL são três ondas senoidais que simulam a medição de tensão da rede. Também poderia ser implementado no lugar da PLL um oscilador na frequência desejada já que na operação isolada não se tem a rede.

\subsection{Controle da tensão na rede}

Para modelagem matemática do sistema do conversor $\mathrm{CC} / \mathrm{CA}$, é utilizado o circuito equivalente representado na figura 4. A tensão chaveada do lado do conversor é modelada como uma fonte de tensão controlada, e o filtro pode ser simplificado por um indutor equivalente em série com a resistência, já que para baixas frequências o capacitor pode ser desconsiderado.

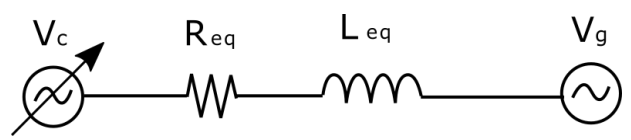

Figura 4. circuito equivalente do conversor CC/CA e filtro

Aplicando as leis de Kirchoff para corrente e tensão e aplicando a transformada de Park podemos obter as equações (3) e (4) do sistema no referencial síncrono:

$$
V_{c d}(s)=R_{e q} I_{d}(s)+s L_{e q} I_{d}(s)+\omega_{g} L_{e q} I_{q}(s)+v_{o d}(s)
$$

$$
V_{c q}(s)=R_{e q} I_{q}(s)+s L_{e q} I_{q}(s)-\omega_{g} L_{e q} I_{d}(s)+v_{o q}(s)
$$
sendo $\omega_{g}=2 * p i * f_{\text {rede }}$ Para a malha de corrente os termos de $v_{o d}$ e $v_{o q}$ podem ser usados numa ação feedforward, e as componentes de $\omega_{g} L_{e q} I_{d}(s)$ e $\omega_{g} L_{e q} I_{q}(s)$ são realimentadas para o desacoplamento entre as duas malhas. Desta forma a função de transferência para a corrente é dada pelas equações (5) e (6):

$$
\begin{aligned}
& \frac{I_{d}(s)}{V_{d}(s)}=\frac{1}{R_{e q}+s L_{e q}} \\
& \frac{I_{q}(s)}{V_{q}(s)}=\frac{1}{R_{e q}+s L_{e q}}
\end{aligned}
$$

Para o controle da tensão no capacitor, escrevendo as equações da corrente obtemos (7) e (8):

$$
i_{l d}-i_{g d}=C_{f} \frac{d v_{o d}}{d t}-\omega C_{f} v_{o q}
$$

$$
i_{l q}-i_{g q}=C_{f} \frac{d v_{o q}}{d t}+\omega C_{f} v_{o d}
$$

Para a malha de tensão é feita a compensação cruzada dos termos $\omega C_{f} v_{o q}$ e $\omega C_{f} v_{o d}$, também considerando a corrente de saída $i_{g}$ como distúrbio, assim a função de transferência para a malha de tensão será dada pela equação (9):

$$
\frac{v_{o_{d q}}}{i_{l_{d q}}}=\frac{1}{C_{f} s}
$$

O objetivo do controle do inversor no modo fonte de tensão é controlar a tensão no capacitor $C_{f}$, fazendo com que esta tensão seja senoidal com a frequência desejada. Figura 5 mostra o diagrama de blocos da estrutura de controle implementada para o inversor. Para a malha de corrente a 
função de transferência em malha fechada considerando o controlador é dada pela equação (10):

$$
\frac{i_{d}}{i_{d}^{*}}=\frac{\left(K_{p}+\frac{K_{i}}{s}\right) * \frac{1}{R_{e q}+s L_{e q}}}{1+\left(K_{p}+\frac{K_{i}}{s}\right) * \frac{1}{R_{e q}+s L_{e q}}}
$$

Aloca-se o zero do controlador de modo a anular o pólo da planta para que a função de transferência em malha fechada seja dado pela equação (11):

$$
\frac{i_{d q}}{i_{d q}^{*}}=\frac{1}{\tau_{i} s+1}
$$

Assim igualando as equações (10) e (11), os parâmetros do controlador podem ser calculado através da expressão (12) (Yazdani and Iravani (2010)):

$$
K_{p}=\frac{L_{e q}}{\tau_{i}} \quad e \quad K_{i}=\frac{R_{e q}}{\tau_{i}}
$$

onde $\tau_{i}$ é a constante de tempo da malha fechada. A malha de corrente é a malha mais rápida do sistema, por isso é escolhido que a frequência de corte $f_{c}$ da malha seja uma década abaixo da frequência de chaveamento de acordo com a equação (13):

$$
\tau_{i}=\frac{1}{2 \pi f_{c}}
$$

Os ganhos do controlador de tensão devem ser calculados para determinada margem de fase $\left(\zeta_{m}\right)$ e frequência de acordo com a equação (14)(Antunes et al. (2016)):

$$
K_{p}=C_{f} \sqrt{\frac{1}{\tau_{i}^{2}} \frac{1-\operatorname{sen}\left(\zeta_{m}\right)}{1+\operatorname{sen}\left(\zeta_{m}\right)}} \quad e \quad K_{i}=\frac{K_{p}}{\tau_{i}} \frac{1-\operatorname{sen}\left(\zeta_{m}\right)}{1+\operatorname{sen}\left(\zeta_{m}\right)}
$$

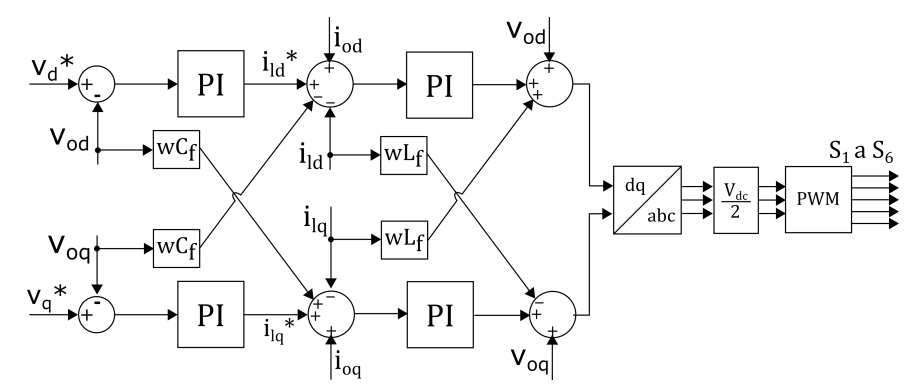

Figura 5. Diagrama de blocos do controle da tensão no modo fonte de corrente.

A malha de tensão foi projetada para margem de fase de $60^{\circ}$ e frequência de corte uma década abaixo da frequência de corte da malha de corrente.

\subsection{Conversor $C C / C C$}

O conversor $\mathrm{CC} / \mathrm{CC}$ é implementado com o objetivo de controlar a transferência de potência para o barramento $\mathrm{CC}$ e controlar a carga e descarga das baterias. O conversor sendo entrelaçado, tem a vantagem de que a corrente total do conversor é dividida entre os indutores, podendo ser dimensionados para valores menores, resultando em redução no tamanho dos componentes. Outro benefício é o aumento da frequência e diminuição da amplitude do ripple da corrente da saída (Assunção (2014)).

Sendo o nível de tensão escolhido para o conjunto das baterias de $144 \mathrm{~V}$ e da tensão no barramento CC de $450 \mathrm{~V}$, o conversor irá atuar em dois modos de operação. O conversor age como um conversor buck (abaixador) ao transferir potência do barramento CC para o conjunto de baterias acionando as chaves superiores e como um conversor boost (elevador) ao transferir potência da bateria para o barramento CC acionando as chaves inferiores.

Para o conversor operando isolado, o controle adotado tem como objetivo controlar a tensão no barramento CC, porque o fluxo de potência é do conjunto de baterias para a rede. A topologia adotada foi o controle em malha fechada da tensão do barramento CC com uma malha externa de tensão e malha interna para controle da corrente dos indutores do conversor $\mathrm{CC} / \mathrm{CC}$.

O modelo do boost pode ser obtido independentemente através da média do modelo de espaço de estados (Erickson and Maksimovic (2007)). Inicialmente é feita o controle das correntes do boost. A função de transferência para o boost considera o barramento CC como uma carga equivalente calculada como a razão do quadrado da tensão do barramento pela potência nominal do conversor. Esta resistência é representado como $R_{c c}$. Sendo $i_{\text {boost }}=i_{L 1}+$ $i_{L 2}$ e desconsiderando a resistência do indutor, a função de transferência da corrente do indutor $\left(i_{\text {boost }}\right)$ pela razão cíclica $(d)$ do boost é dada por (15):

$$
\frac{i_{\text {boost }}(s)}{d(s)}=\frac{2 V_{b b}}{(1-D)} \frac{s R_{c c} C_{c c}+2}{s^{2} R_{c c} L C_{c c}+s L+R_{c c}(1-D)^{2}}
$$

Também pelo modelo de espaço de estado temos a função de transferência da tensão no capacitor do link cc pela corrente, dado pela equação (16)

$$
\frac{v_{c}(s)}{d \hat{(s)}}=\frac{V_{b b}}{(1-D)^{2}} \frac{-L s+2 R_{c c}(1-D)^{2}}{s^{2} R_{c c} L C_{d c}+s L+2 R_{c c}(1-D)^{2}}
$$

Como a corrente é responsável por manter a tensão do barramento CC, a função de transferência da malha de tensão deverá ser $V_{d c}(s) / i_{\text {boost }}(s)$, que é obtida pela razão das equações 15 e 16

$$
\frac{v_{c}(s)}{I_{l} \hat{(s)}}=\frac{1}{(1-D)} \frac{-L s+2 R_{c c}(1-D)^{2}}{2\left(s R_{c c} C_{c c}+2\right)}
$$

A malha de controle do conversor $\mathrm{CC} / \mathrm{CC}$ pode ser visto na figura 6 .

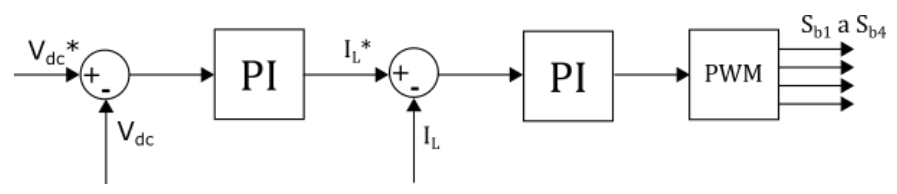

Figura 6. Diagrama de blocos do controle da tensão no barramento DC

Os ganhos dos controladores podem ser encontrados pelo método da resposta em frequência, na qual os controladores são projetados para terem uma década de diferença da frequência de chaveamento.

A performance da bateria não irá ser considerada neste artigo. O conjunto de baterias foi aproximado por uma fonte de tensão DC ideal.

\section{SIMULAÇÃO}

O conversor é implementado no Matlab/Simulink. Os parâmetros do sistema foi apresentado na tabela 1. Os 
sensores de tensão e corrente são considerados com ganho unitário. Na figura 7 mostra a tensão no PAC com o conversor sem carga. Sem carga, o inversor é capaz de sintetizar no PAC a tensão de referência.
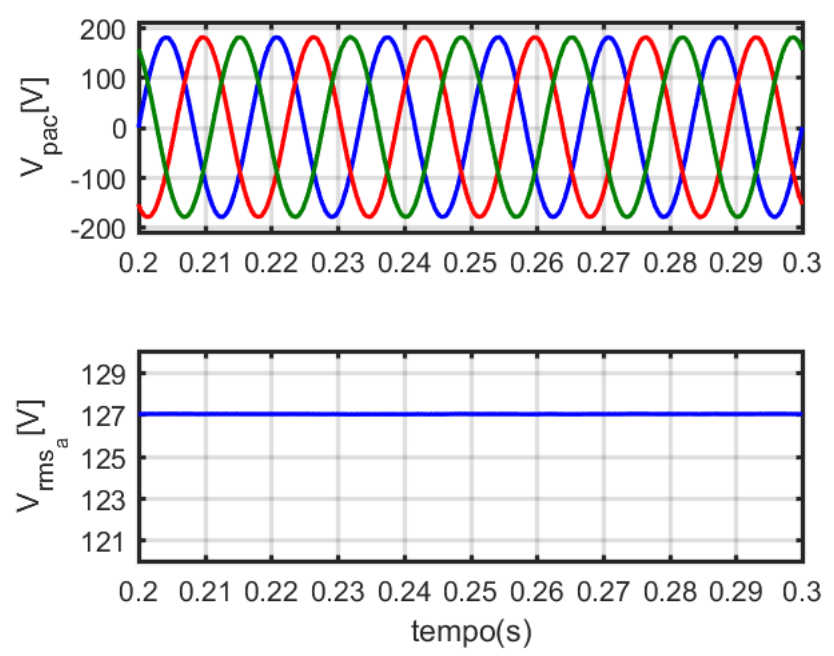

Figura 7. a)Tensão trifásica no PAC, b) tensão RMS da fase $\mathrm{A}$
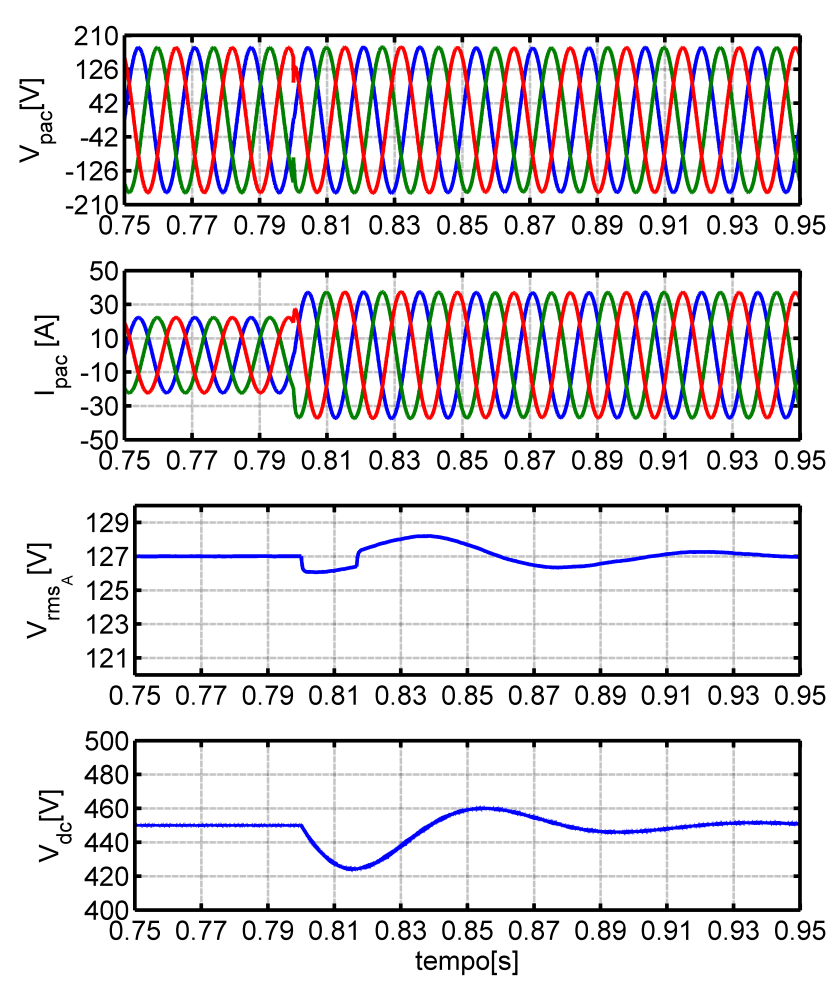

Figura 8. a)Tensão trifásica no PAC, b) corrente trifásica no PAC, c) tensão RMS na fase A, d) tensão no barramento CC

Carga Linear As figuras 8 e 9 mostram o resultado da performance do conversor quando conectado a uma carga linear. Inicialmente é aplicada uma carga de $6 \mathrm{~kW}$. No tempo $\mathrm{t}=0.8 \mathrm{~s}$ a carga é aumentada em $4 \mathrm{~kW}$, sendo a

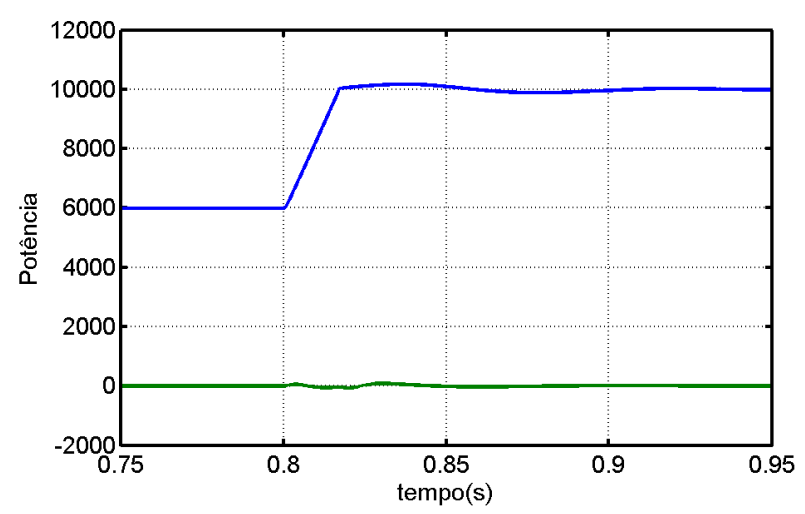

Figura 9. Potência medida no PAC

carga $100 \%$ da potência do conversor. Na figura 8, temos a dinâmica da tensão e corrente no PAC e tensão no barramento CC. No momento do degrau de carga, a tensão de pico no PAC cai $1 \mathrm{~V}$ e demora 6 ciclos para retornar ao valor de referência. Na figura 9, temos a potência no PAC, o sinal positivo na potência indica que o inversor fornece potência ativa. Sendo a carga linear não há distorção na tensão. Na corrente a distorção encontrada foi de 1,22\%.

Carga não-linear Na figura 10 temos o comportamento do inversor quando há uma carga não-linear conectada ao PAC. A carga linear é um retificador trifásico com filtro e uma carga resistiva. O inversor consegue estabelecer a tensão no PAC com valor de rms de $127 \mathrm{~V}$, mas a tensão possui harmônicos. A tensão da rede possui harmônicos de quinta e sétima ordem característicos de uma retificação trifásica. A distorção da corrente é $18.9 \%$ e na tensão de 13.2\%. A ANEEL no documento "Procedimentos de Distribuição de Energia Elétrica no Sistema Elétrico Nacional PRODIST", módulo 8, estabelece que os níveis de distorção harmônica total da tensão no nível menor que $1 \mathrm{kV}$ deve ser de $10 \%$ para conexão com a rede (ANEEL (2018)). Usando este documento como referência de qualidade de energia, o nível de harmônicos da tensão está acima do recomendado. O controle PI em referencial síncrono apenas consegue o erro zero para a componente DC, sendo ineficaz para o controle de harmônicos (Liu et al. (2013)). Na figura 11 , temos a potência consumida pela carga não linear. $\mathrm{O}$ formador de rede fornece $8 \mathrm{~kW}$ de potencia ativa e $2 \mathrm{kVAr}$ de potência reativa. O conversor é capaz de fornecer tanto potência ativa quanto reativa devido ao armazenador de energia existente no sistema. Apesar da tensão do barramento $\mathrm{CC}$ ser controlada pelo conversor $\mathrm{CC} / \mathrm{CC}$, pode perceber na figura 10 que a tensão do barramento $\mathrm{CC}$ tem uma ondulação vinda da carga não linear, o que mostra que os conversores não são completamente desacoplados.

\section{CONCLUSÃO}

Neste trabalho foi implementado um inversor funcionando como fonte de tensão para aplicação como formador de rede em microrredes. Foi implementado o controle da tensão do PAC em referencial síncrono com o controle do barramento CC pelo conversor bidirecional e controle da corrente do boost.

Na simulação executada o sistema mostrou que consegue alcançar o objetivo principal, nas duas condições de carga 

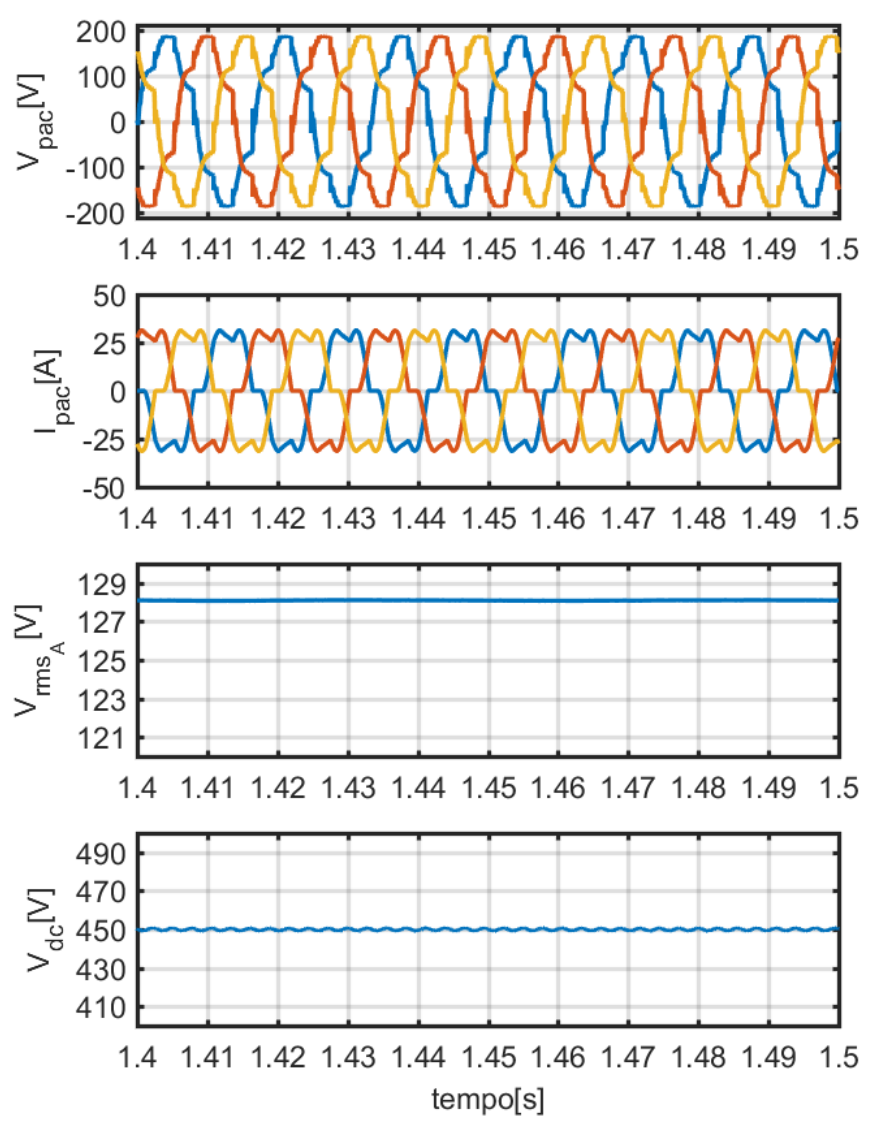

Figura 10. a)Tensão trifásica no PAC, b) corrente trifásica no PAC, c) tensão RMS na fase A, d) tensão no barramento $\mathrm{CC}$

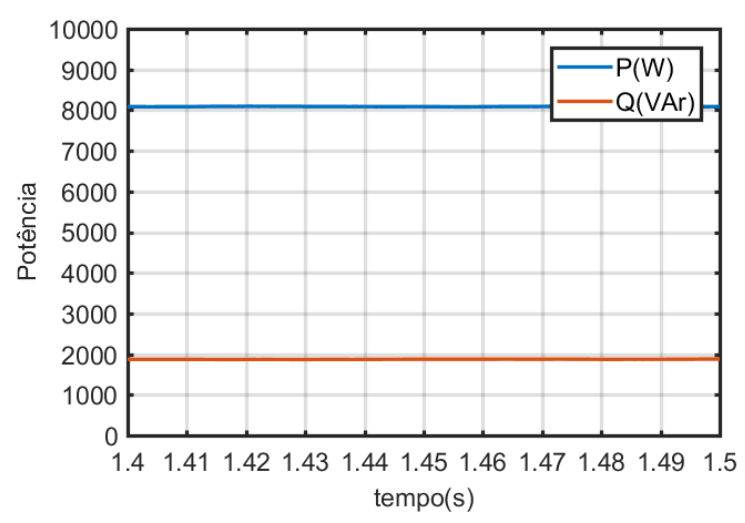

Figura 11. Potência medida no PAC

e sem carga. O inversor consegue estabelecer no ponto de acoplamento comum a tensão de referência. O barramento CC também permaneceu com a tensão controlada no nível de referência. Analisando os resultados encontrados na simulação, pode-se afirmar que o inversor cumpre a função de formador de rede ao estabelecer uma tensão com valor RMS e frequência controlada no PAC. Em condições de carga linear, o inversor obteve resultados satisfatórios mesmo quando há uma mudança no valor da carga. Em condições de carga não linear, o inversor deixa a desejar já que não cumpre os requisitos da norma para o nível de harmônicos na tensão. Para melhoria do projeto recomenda-se implementar o controle de harmônicos e de desbalanço.

\section{REFERÊNCIAS}

ANEEL (2012). Resolução normativa 482. Estabelece as condições gerais para o acesso de microgeração $e$ minigeração distribuída aos sistemas de distribuição de energia elétrica, o sistema de compensação de energia elétrica, e dá outras providências.

ANEEL (2018). Procedimentos de distribuição de energia elétrica no sistema elétrico nacional prodist - módulo 8 - qualidade da energia elétrica.

Antunes, H., Silva, S., and Cardoso Filho, B. (2016). Análise e operação de uma microrrede de energia elétrica. In Simpósio Brasileiro de Sistemas Elétricos,.

Araújo, L.S. et al. (2017). Controle de conversores em microrredes autônomas com sistemas de armazenamento distribuídos. Dissertação de Mestrado. Faculdade de Engenharia Elétrica e de Computação, Universidade Estadual de Campinas,.

Assunção, M.R. (2014). Estudo e implementação de um conversor cc-cc boost entrelaçado em regime de condução contínua. Dissertação de Mestrado. Programa de Pós-Graduação em Engenharia Elétrica, Universidade Federal de Minas Gerais (UFMG).

Bian, J., Zang, C., Li, X., Hu, B., and Gu, W. (2017). The rapid development of a three-phase grid-forming micro-source inverter. In 2017 2nd International Conference on Power and Renewable Energy (ICPRE), 51-55. IEEE.

de Matos, J., Ribeiro, S., e Silva, F., et al. (2014). Controle da potência gerada em microrredes autônomas e isoladas com fontes de energia renováveis e sistema de armazenamento com bancos de baterias. Rev. Eletrônica Potência, 19(2), 152-162.

Erickson, R.W. and Maksimovic, D. (2007). Fundamentals of power electronics. Springer Science \& Business Media.

Kahlane, A., Hassaine, L., and Kherchi, M. (2014). Lcl filter design for photovoltaic grid connected systems. the Journal of Renewable Energies, 227-232.

Liu, Q., Tao, Y., Liu, X., Deng, Y., and He, X. (2013). Voltage unbalance and harmonics compensation for islanded microgrid inverters. IET Power Electronics, 7(5), 1055-1063.

Rocabert, J., Luna, A., Blaabjerg, F., and Rodriguez, P. (2012). Control of power converters in ac microgrids. IEEE transactions on power electronics, 27(11), 47344749.

Serban, I. and Ion, C.P. (2017). Microgrid control based on a grid-forming inverter operating as virtual synchronous generator with enhanced dynamic response capability. International Journal of Electrical Power \& Energy Systems, 89, 94-105.

Singh, B., Mishra, S., et al. (2018). Dual mode operational control of single stage pv-battery based microgrid. In 2018 IEEMA Engineer Infinite Conference (eTechNxT), $1-5$. IEEE.

Teodorescu, R., Liserre, M., and Rodriguez, P. (2011). Grid converters for photovoltaic and wind power systems, volume 29. John Wiley \& Sons.

Yazdani, A. and Iravani, R. (2010). Voltage-sourced converters in power systems: modeling, control, and applications. John Wiley \& Sons. 